\title{
The Universal Grand Unified Theory Based on Life and Thought
}

\author{
Jinzhong Yan \\ Changsha Jiage'er Machinery Manufacture Co., Ltd., Pingtang Town, Changsha City, Hunan 410208, China
}

\begin{abstract}
The universe is life, which includes galaxies, living beings, society and man-made worlds. The universe is unified in life and thought. The universal grand unified theory is a theory to completely unify all phenomena in the universe with a fundamental mode (structural mode, operational mode, generation mode and evolution mode). The book of changes of China: The great ultimate two states - four images - eight hexagrams are life science and thought science theories, which represent the fundamental mode of universal unification, i.e. represent 1) structural mode, operational mode, generation mode and evolution mode of life; 2) the four states that life has simultaneously: 0 dimension, 1 dimension, 2 dimension, 3 dimension, and these four states have different structural and operational modes respectively; 3) the breathing and four seasonal trajectories of celestial bodies; 4) three generation modes of life: generation by transformation, generation by augmentation, and self-generation; 5) five dynamics systems inside life; 6) the sublimation and degration of life in the universe, the unification of religion and science; 7) the classification mode of the universal being; 8) the derivation mode of life; 9) the transformation between different forms of life in the universe; 10) the unification of six grand forces in the universe. All life systems in the universe: galaxy, organism, society, man-made worlds as well as cognitive science, natural science, social science are all in conform with the mode of the book of changes.
\end{abstract}

Key words: The book of changes, life, thought, universe, grand unified theory.

\section{Introduction}

Modern scientists have been striving to build a grand unification theory that encompasses all beings in the universe to solve all the problems such as the creation of the universe and life. But since modern science is still at the stage of representation science and individual science \& technology [1], and has little research on the most important phenomenon in the universe: life and thought, so their endeavors are always fruitless.

The universe is life, which has been thoroughly studied by traditional Chinese science: The Book of Changes, the Taoist, the Buddhist and traditional Chinese medicine, and formed complete life universe theory. There are also lots of clear discourses in Taoist classics, Buddhist classics and traditional Chinese medicine classics [2-5]. Traditional Chinese science

\footnotetext{
Corresponding author: Jinzhong Yan, senior engineer, research field: disciplinary sciences. E-mail: yanjinzhong32@sina.com.
}

belongs to cultivation type of life science and cognitive science [6]. And modern science has not carried out substantive research on life and thought.

The universe is inherently unified. The dominating movement of the universe is the movement of four seasons and movement of thought [7]. The universe is a living entirety, comprising various kinds of matters and systems. Modern science has only researched the appearance and individuals of the cosmos, while traditional Chinese science stresses on the study of the universal living entirety and thought. The sum of these two has formed a complete research of the universe.

So the unification of two major scientific system from the East and West forms the universal grand unified theory, the overall thought from the East is the core of the universal grand unified theory. Physics is unified in life science and modern science is unified in traditional Chinese science. The book of Changes, as the core of traditional Chinese science [8], encompasses the cosmos and their movements and 
changes, and is the unified mode of the universe.

\section{What is the Universal Grand Unified Theory?}

As its name suggests, the universal grand unified theory is a theory to completely unify all phenomenon in the universe and their movements and changes. Specifically, it is a theory to unify all phenomena in the universe with a basic mode (structural mode, operational mode, generation mode and evolution mode).

The basic mode of universal grand unification covers: 1) the structural mode, operational mode, generation mode and evolution mode of galaxies, living beings, society, man-made worlds in the universe; 2) Conversion between different lives, i.e. conversion between galaxy life, living being, society and man-made worlds.

According to this mode, the universal grand unification includes: 1) unification of cognitive science; 2) unification of time \& space [9]; 3) unification of physics and biology [1]; 4) unification of the cosmos (galaxies, living beings, society, man-made worlds) and unification of all forces in the universe [7]; 5) unification of religion and science; 6) unification of two scientific systems from the East and the West [6]; 7) structure of science and unification of the science; 8) unification of all human knowledge.

The unification of four major forces [10] by physicists does not conform with the requirements of the universal grand unification theory.

\section{The Universe is Unified in Life and Thought}

Life in universe includes galaxies, living beings, society and man-made worlds. They all have four seasonal structure and movements. And all matters in the universe are four seasonal life matters [11], and are subject to four seasonal rules.

Life has thought. Thought movement is another important movement of life, which are mainly sublimation and degration movements. Life is classified into four major states: 0 dimension, 1 dimension, 2 dimension, and 3 dimension [9]. The dimension of life is decided by whether the life has desire or not [3]. Those without desire go up to 2-dimension, 1-dimension and 0-dimension, while those with desire go down to 3 dimension. Life is the contradictory unity of mind and matter, and mind and matter is constantly interacting with each other and converting into each other. The sublimation and degration of life is constantly happening and the two sides are in a dynamic equilibrium.

The four season movement and thought movement are the most important movement of the universe. The universe is unified in life and thought. The universal grand unification theory should be based on life and thought. Life has four seasonal structures, four seasonal movements, four major states and sublimation and degration conversions between the four major states simultaneously. The universal grand unification mode should have this characteristic, while The Book of Changes of China has exactly this characteristic.

\section{The Universal Unification Mode: The Book of Changes}

Traditional Chinese science: The Book of Changes , Taoist, Buddhist, Traditional Chinese medicine belongs to life science and cognitive science, with The Book of Changes as its climax [8]..

\subsection{The Unification Mode of The Book of Changes}

The Book of Changes is a theory of life science and cognitive science. It shows four seasonal structures, four seasonal movements, four major dimensions and sublimation and degration conversions between the four major dimensions and is the unification mode of the cosmos. By integrating The Book of Changes , Taoist, Buddhist, Traditional Chinese Medicine and this writer's research [4, 8, 9, 12-15], we have the unified mode of The Book of Changes as the following Table 1. 
Table 1 The unified life mode of The Book of Changes.

\begin{tabular}{|c|c|c|c|c|c|c|c|c|}
\hline \multirow{2}{*}{$\begin{array}{l}\text { Universe } \\
4 \text { status }\end{array}$} & \multicolumn{2}{|c|}{ The Book of Changes } & \multirow{2}{*}{$\frac{\text { Taoist }}{4 \text {-status }}$} & \multirow{2}{*}{$\frac{\text { Buddhist }}{4 \text { status }}$} & \multicolumn{3}{|c|}{ Man's System and Dimension } & \multirow[t]{2}{*}{$\begin{array}{l}\text { Method of } \\
\text { Generation } \\
\end{array}$} \\
\hline & 4 status & $\begin{array}{l}\text { Number of } \\
\text { seasons }\end{array}$ & & & Structure & $\begin{array}{l}\text { Dynamic } \\
\text { system }\end{array}$ & Energy & \\
\hline 0 dimension & Great ultimate & 0 & Тао & $\begin{array}{l}\text { The purified } \\
\text { realm of } \\
\text { Buddha }\end{array}$ & Brain & Thought & $\begin{array}{l}\text { Thought } \\
\text { energy }\end{array}$ & No birth \& death \\
\hline 1 dimension & 2 status & 2 & one & $\begin{array}{l}\text { The colorless } \\
\text { realm }\end{array}$ & Heart \& lung & Breathe & $\begin{array}{l}\text { Breathing } \\
\text { energy }\end{array}$ & $\begin{array}{l}\text { Generation by } \\
\text { transformation }\end{array}$ \\
\hline 2 dimension & 4 images & 4 & two & $\begin{array}{l}\text { The color } \\
\text { realm }\end{array}$ & $\begin{array}{l}\text { Liver, spleen, } \\
\text { kidney }\end{array}$ & $\begin{array}{l}\text { Blood making } \\
\text { and } \\
\text { transportation }\end{array}$ & $\begin{array}{l}\text { Blood } \\
\text { energy }\end{array}$ & $\begin{array}{l}\text { Generation by } \\
\text { augmentation, and } \\
\text { self-generation }\end{array}$ \\
\hline 3 dimension & $\begin{array}{l}8 \text { hexagrams (64 } \\
\text { hexagrams) }\end{array}$ & $8(64)$ & three & $\begin{array}{l}\text { The realm of } \\
\text { desire }\end{array}$ & Body & $\begin{array}{l}\text { Biochemical } \\
\text { reaction }\end{array}$ & $\begin{array}{l}\text { Chemical } \\
\text { energy }\end{array}$ & $\begin{array}{l}\text { Generation by } \\
\text { augmentation, and } \\
\text { self-generation }\end{array}$ \\
\hline
\end{tabular}

4.2 Interpretation of the Book of Changes: the Universal Unification Mode

4.2.1 Four Major Status Exist in Life Simultaneously

According to Table 1, The Book of Changes talked about four dimensions, while life has four corresponding dimensions, i.e. four dimensions exist in life simultaneously. And the four major statuses include four major status of life: four major status of the spiritual system; four major status of the information system; four major status of the dynamic system, four major status of the structural system etc.

\subsubsection{The Four Major Dimensions Have Four} Seasonal Structures and Movements

In The Book of Changes, different dimensions have different four seasonal structures and movements. The great ultimate is 0 dimension, which is the highest status in the universe, and is not subject to four seasons and has no birth or death, i.e. so called "outside of the three Realms, not inside the five Xings" by the Buddhist \& Taoist. The two states have two seasons, for example, the earth's North Pole area has only two seasons a year - day time for half a year and night time for half a year. The four images have four seasons and this is the four seasonal movements that are very familiar to everyone. The eight hexagrams have eight seasons. We add the two solstices, two equinoxes, plus the four beginnings and then a year has eight seasons. The 64 hexagrams have 64 seasons, dividing a year into 64 feature points, i.e. 64 seasons.

In life, there are the structure and movement of great ultimate, two status, four images, eight hexagrams and 64 hexagrams simultaneously [14]. Four states exist inside life simultaneously. Different status have different operational mode, which is guided by the dominant status. When life is in three dimensional status, life operates in complicated 8 seasons or 64 seasons in accordance with 8 hexagrams - 64 hexagrams. When life is in two dimensional states, life operates in four seasons in accordance with four images. When life is in one dimensional status, life operates in two seasons in accordance with two states. 3 dimension, 2 dimension, 1 dimension all have the movement of life and death. When life is in zero dimensional status, life has no movement of life and death.

4.2.3 The Breathe and Four Seasonal Trajectory of Celestial Bodies Described in The Book of Changes

The four seasonal movement is the breathing movement [7], whose characteristics of movement is the elliptical orbit of celestial bodies. For example, the Earth's orbit around the Sun is elliptical and it is the breathing movement. In spring and summer, it is exhalation; in autumn and winter, it is inhalation. The Moon's orbit around the Earth is also elliptical. The Earth is an ellipsoid, and the Earth's rotation is also 
the breathing movement.

The elliptical orbit is the great ultimate. The spring and summer is Yang, the autumn and winter is Yin and these are the two states. The two solstices plus the two equinoxes are four images. The two solstices plus the two equinoxes plus the four beginnings are the eight hexagrams. It is possible to find 64 feature points that correspond to 64 hexagrams. The Moon's elliptical orbit around the Earth is also the great ultimate -64 hexagrams diagram [15] .

4.2.4 The Book of Changes Shows Generation Mode of Different Life and Conversion between Different Lives

The Book of Changes shows four generation mode of life: no birth and death; generation by transformation, generation by augmentation, and self-generation [3]. The great ultimate is the master of the cosmos, which has no birth and death. The universal thought organizes life material automatically into galaxy life [7], so the generation of galaxies are generation by transformation. Generation by transformation is the automatic organization of life, which comes into being out of nothing. Galaxies, as life, have internal female/male mechanisms mechanism of change and mechanism of stability and these two copulate to generate the life energy to nurture itself and living being, so it's called generation by augmentation. Unlike living beings, galaxies do not generate individuals that resemble themselves. But living beings can generate individuals that resemble themselves, so it's called self-generation.

The female/male mechanisms of the solar system and Earth life copulate to cause generation by augmentation and produce various kinds of biological macromolecules needed to product life and the four seasonal structure of life, producing living beings under the automatic organization of the life information body from galaxies in the universe [16]. So the origin of life on Earth was generation by transformation. Society, man-made objects are produced under the automatic organization of human thought, so it is generation by transformation.

Thus, life in the universe: galaxy life, living being, society, man-made worlds are converted through different generation method, i.e. the universal thought and universal life matter form celestial bodies through automatic organization, the life information of celestial bodies automatically organizes the life matters of generation by augmentation in the solar system to form living being, humans form society through automatic organization, and automatic organization by humans create man-made worlds.

4.2.5 The Book of Changes Shows the Five Dynamic Systems of Life

The four major states of The Book of Changes shows the four major dynamic system inside life: the movement of thought \& information flow, the breathing movement, the heart pulse and active transportation, the interaction and active, automatic chemical \& physical reactions between the mechanism of change, mechanism of stability and mechanism of control. Life as a whole has internal closed-loop circuit (particles flow) and energy flow. So The Book of Changes shows the five dynamic systems of life [7].

4.2.6 The Book of Changes Shows the Sublimation and Degration of Life in the Universe, and the Unification of Religion and Science

Life in the universe is classified into four states: 3 dimensional person, 2 dimensional person, 1 dimensional person, and 0 dimensional person [9]. The 0 dimensional person is god, the 3 dimensional person is human. The 2 dimensional person and 1 dimensional person are in the middle. The sublimation of life means life's evolution from low energy to high energy (reverse evolution). The cultivation of the Taoists, Buddhists is the transformation from 3 dimensional life of low energy to 2 dimensional life, 1 dimensional life and 0 dimensional life of high energy. This reverse evolution of life is from self-regeneration to generation by augmentation and generation by transformation, until finally reach no birth and death; 
The reverse evolution is the sublimation of spirit and is the thought movement of no desire.

The degration of life means life's evolution from high energy to low energy (forward evolution). It means 0 dimensional life, 1 dimensional life and 2 dimensional life of high energy degrades to 3 dimensional life of low energy. The forward evolution is the degration of the spirit, and is the result of desire.

Life is the entity of contradiction between spirit and matter. Persons can be cultivated to god through spiritual sublimation, but also can degrade to humans or even lower due to desire. This means man and god can transform to each other.

The 3 dimensional persons have lost divinity, so they understand the physical world, build materialistic science \& technology. After losing divinity, they cannot understand god and theology, but they still revere god, so they pick up the lost theology as faith and formed religion. When modern science moves forward to unlock the mystery of the universe and god, humans begin to re-understand god, give up physical desire and sublimate their spirit, humans will return to the dominion of god.

So, The Book of Changes also shows the unification of theology and science, and the unification of religion and science.

4.2.7 The Book of Changes Shows the Classification of the Cosmos

Longitudinally, The Book of Changes divides things into four dimensions. Laterally, all dimensions of The Book of Changes have structures. The great ultimate, being three things, includes three as one. The two states are divided into two types of things: positive \& negative. The four images are divided into four types of things and the eight hexagrams are divided into eight types of things. The sixty-four hexagrams are divided into 64 types of things [14].

4.2.8 The Book of Changes Shows the Derivation Mode of Life

The derivation mode of The Book of Changes is one divided into two (forward evolution), two combined into one (reverse evolution). Cell division [16] is one divided into two. And the Taoist's cultivation in accordance with The Book of Changes is a procedure of two combined into one [3].

4.2.9 The Book of Changes Shows the Unification of Six Grand Forces in the Universe

In The Book of Changes, the great ultimate means "so big that it has no outside, so small that it has no inside", with permeability, that's equivalent to neutrinos, and is a weak interaction force. The two status mean straight line coordinates, i.e. $-\longrightarrow$, which means the Yin \& Yang confinement, i.e. quark confinement $[9,16]$. The four images constitute plane coordinates, i.e.

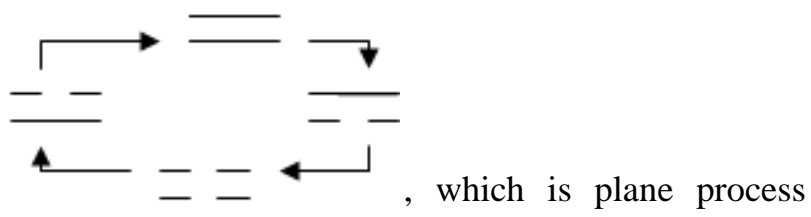
and means the strong interaction [9, 16]. The eight hexagrams constitute three dimensional coordinates, which means three dimensions and bond. There are electric-magnetic forces between quark confinement, the strong interaction and bond force. The great ultimate - two states - four images - eight hexagrams as a life entity has thought, and the force of thought is universal gravitation and universal repulsion. So The Book of Changes shows the unification of six grand forces in the universe [7].

\section{How to Understand and Construct the Universal Grand Unification Theory: Unified Cognitive Science}

The universe is inherently unified and the universal grand unified theory is an objective existence. Man has the power to understand the cosmos and construct the universal grand unified theory.

\subsection{Methods and Means to Understand the World}

5.1.1 Man's Cognitive System and Four Seasonal Structure

Man as the subject of cognition, has the power to 
under the cosmos. Man has cognitive system, with brain as its center. Eyes, ears, nose, tongue, body and sense are man's sensory organs. Human cognitive system includes inner sensory system and outer sensory system [18]. Human cognitive system has four seasonal structures and four seasonal movement, i.e. perception of information, treatment of information and formation of conclusion, storage of results, information output.

\subsubsection{Cognitive Objects}

These include celestial bodies, living beings, society, man-made worlds, all matters - visible matters and invisible matters, visible lives and invisible life that make up the universe. All cognitive objects are lives or life matter.

5.1.3 The Occurrence of Cognition - Interaction between Cognitive Organs and Cognitive Objects

Human cognitive organs can sense all characteristics of the cosmos. Complete correspondence is formed between cognitive organs and cognitive objects. When cognitive organs interact with characteristics of objects, cognition happens.

\subsection{The Deepening of Cognition and Improvement of Cognitive Ability}

To deepen the understanding of things, it is needed to improve the cognitive ability of man. The East and the West show completely different method in this respect and formed completely different scientific systems.

5.2.1 The High Performance of Human Cognitive Organs and Oriental Science \& Technology System

The oriental science is method of inward pursuit [3]. By means of cultivation to improve inner energy of human body, enable the high performance of the cognitive system on the body and understand the cosmos via body of high performance. The cognition of oriental science is completely intuitive and it is the accurate cognition of the status of things. Since the cultivations of the Taoist and Buddhist have reached the highest status (dimension) of the universe, so they can and already have understood the cosmos and their rules, establishing the oriental science \& technology systems that center on The book of changes, Taoist, Buddhist, traditional Chinese medicine. This system belongs to cultivation type of life science and thought science [6].

5.2.2 The Materialization of Cognitive Organs and Modern Science \& Technology System

The western science is method of outward pursuit [3], which extends human cognitive organs and system outwards by means of matters and the instrumentation of cognitive organs and systems. For example, telescope and microscope are the materialization of human vision, etc. By means of the expression and improvement of human cognitive organs with instruments, they improved human cognitive ability, deepened understanding on things and formed modern science. Until now, modern science is still restricted in the superficial, individual and local study of the cosmos, and has little understanding on life whole. It belongs to the system that studies and uses matters [6].

\subsubsection{Four Major Dimensions of Cognitive Ability}

Based on the inside energy of human body, the oriental science divides cognitive ability into four dimensions: 3 dimension, 2 dimension, 1 dimension, 0 dimension [9]. People of different dimensions understand different objects. Only in the status of Buddha and Tao, it's possible to accurately understand the cosmos.

Modern scientific detectors still belong to physical detectors that cannot detect life and thought. Physical detectors can also be divided into four dimensions: 3 dimension - appearance detector, 2 dimension - inside status detector, 1 dimension - high energy particles detector, 0 dimension - neutrino detector. And human quality is shown as intelligence of different dimensions.

\subsection{Future Life and Thought Detector}

Once modern science joins hands with Oriental 
science to enter the stage of life science and thinking science at which it's able to make inorganic life material and inorganic life body [1], man will be able to manufacture life and thought detector and the understanding of the universe by modern science will quickly draw close to oriental science. Materialized life and thought detector in the future will also be divided into four dimensions: 3 dimension, 2 dimension, 1 dimension and 0 dimension.

\section{Unified Cosmos: Life Cosmos}

The universe is life, whose dominant movements are four seasonal movements and thinking movements.

\subsection{Unlimited Universe and Permanent Four Seasonal Movements}

The solar system is a four seasonal entity [1]. Galaxies, galaxy clusters, super galaxy clusters and super giant galaxy clusters are also four seasonal entities. The functional unit of the universe is galaxy. In galaxy clusters, each galaxy performs its own function and contributes to the overall function of the galaxy cluster. All galaxies in galaxy clusters carry out relative movement and interaction in accordance with four seasonal rules. For example, the local cluster which the Milky Way belong to, from the Milky Way's perspective, some galaxies move towards the Milky Way and some galaxies move away from the Milky Way. This is the need of four seasonal movement of galaxies, and not the expansion of the universe.

In the universe, small scale four seasonal celestial bodies participate in the four seasonal movement of large scale four seasonal celestial bodies. The universe is always in dynamic four seasonal equilibrium. The universe is unlimited. The universe is constantly evolving in the never-ending four seasonal circulation process in which one thing is born, another thing is growing. And the interactions between all galaxies in the universe are bound to form the microwave background of the universe.

6.2 Four Major Dimensions and Sublimation/Degration of Galaxies in the Universe

Galaxies in the universe are divided into four dimensions: 3 dimensional galaxy - spiral galaxy, 2 dimensional galaxy - elliptical galaxy, 1 dimensional galaxy - quasar, and 0 dimensional galaxy - neutrino field [9]. Galaxies in the universe transform from 3 dimension, 2 dimension to 1 dimension, 0 dimension collectively under the active control of thinking as well as breathing movement [7]. Then they drop to 3 dimension and start the next circulation.

\subsection{Life is Inherent in the Universe}

Galaxies are four seasonal life [7]. The movement and transformation of the universe is the movement and transformation of life. Life is inherent in the universe, so the question of the origin of the universe and life simply does not exist.

\subsection{Living Being is the Reproduction of Celestial Bodies}

Living beings reflect the universe and different living beings reflect different universes. The ecosystem of the Earth belongs to reflective ecosystem of the universe [7]. Galaxies have innate reproduction structures. Life on Earth can produce all kinds of biological molecules, and four seasonal structure of all living beings. The cognitive body information released by celestial bodies uses all kinds of biological molecules and four seasonal structure of all living beings on earth to produce living being by means of automatic organization [16].

\subsection{The Sublimation/Degration of Life on Earth}

The status of man sublimates/degrades between 3 dimension, 2 dimension to 1 dimension, 0 dimension. It sublimates in case of no desire and cultivation. It degrades in case of desire and corruption. Meanwhile, the inner energy of the entire solar system can affect 
the improvement of the inner energy of human body. When the solar system as a whole is in spring and summer, the inner energy of human body can be easily raised and man and ecosystem sublimate. When the solar system as a whole is in autumn and winter, the inner energy of human body can hardly be raised, man and ecosystem degrade. According to the Mayas, the solar system conducts four seasonal movement orbiting the Pleiades and on December 21, 2012, mankind ended the last solar calendar, ended the low energy era of the solar system, and began a new solar calendar, i.e. the inner energy of the solar system will be raised gradually and the inner energy of life on Earth, especially the inner energy of man will be easily raised [19]. This will have large impact on humans.

\section{Unification of Man, Nature, Society and Man-Made Worlds}

Man is a product of nature and the automatic organization of human forms society, forms the four seasonal structure and four seasonal movement of the society, and create physical world and spiritual world. In society, human quality is the decisive factor. Human quality is the result of movement of the universe, and the result of human cognitive actions. People of different quality understand different status of the universe, and create different physical world and spiritual world. The universe has dimensions, as well as man and the society. Man, nature, society and man-made worlds form an entity of mutual interaction and mutual affection.

\subsection{The Four Seasonal Structure and Four Seasonal Movement of the Society}

The four seasonal structure of the society includes the discovery system, innovation system, consumption application system, quality improvement system and social control system. The four seasonal structure of the society includes the four seasonal structure of change mechanism and stable mechanism, i.e. governmental four seasonal structure and people's four seasonal structure. The two four seasonal systems take nature, man and society as their cognitive objects, and transform the cognitive results into four seasonal movement (Fig. 1) [20].

Aside from the transverse four seasonal structure and four seasonal movement of the society, the society also undergoes four seasonal development longitudinally, and develops forward in a spiral way.

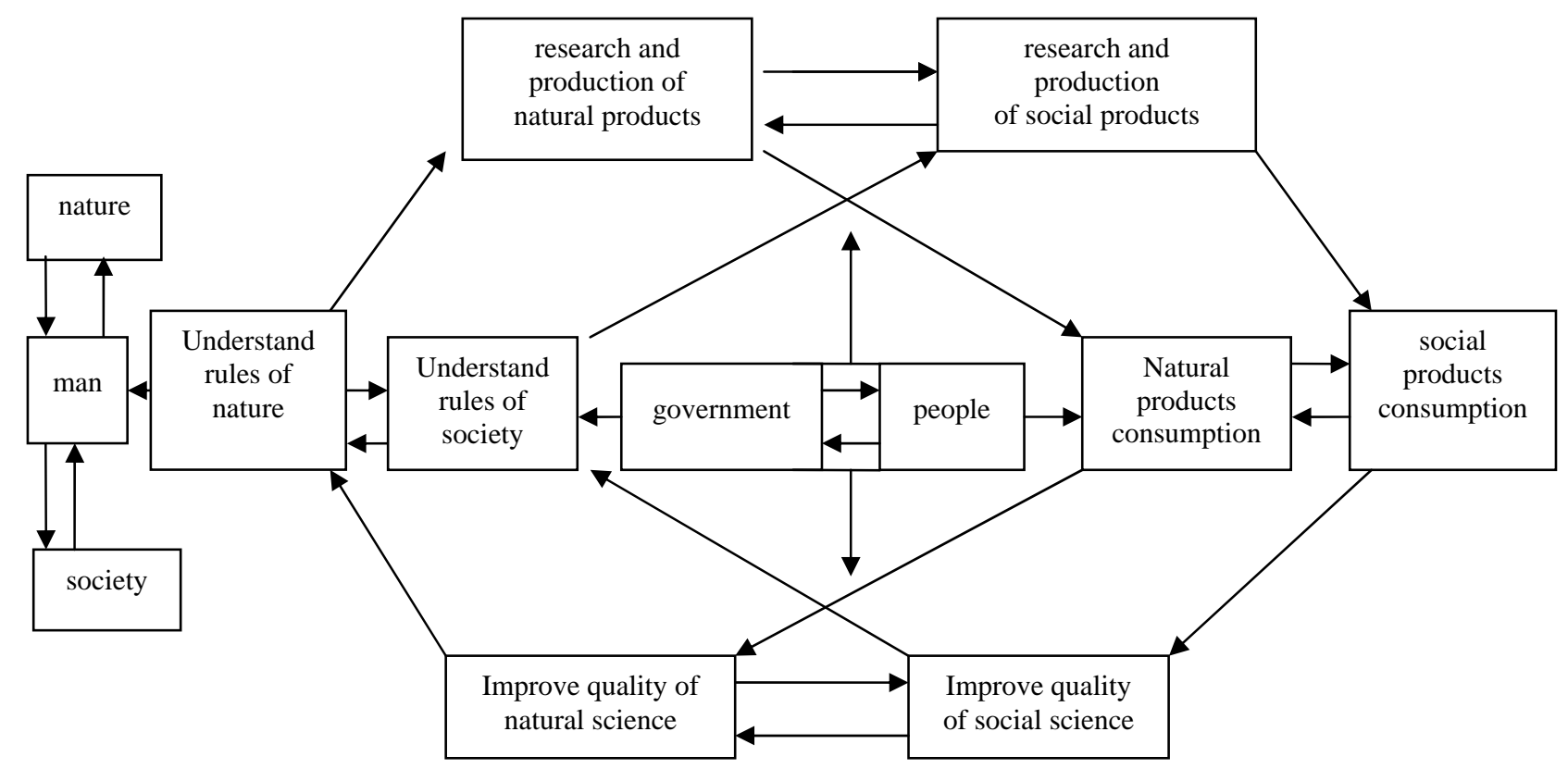

Fig. 1 The four seasonal structure and four seasonal movement of the society. 


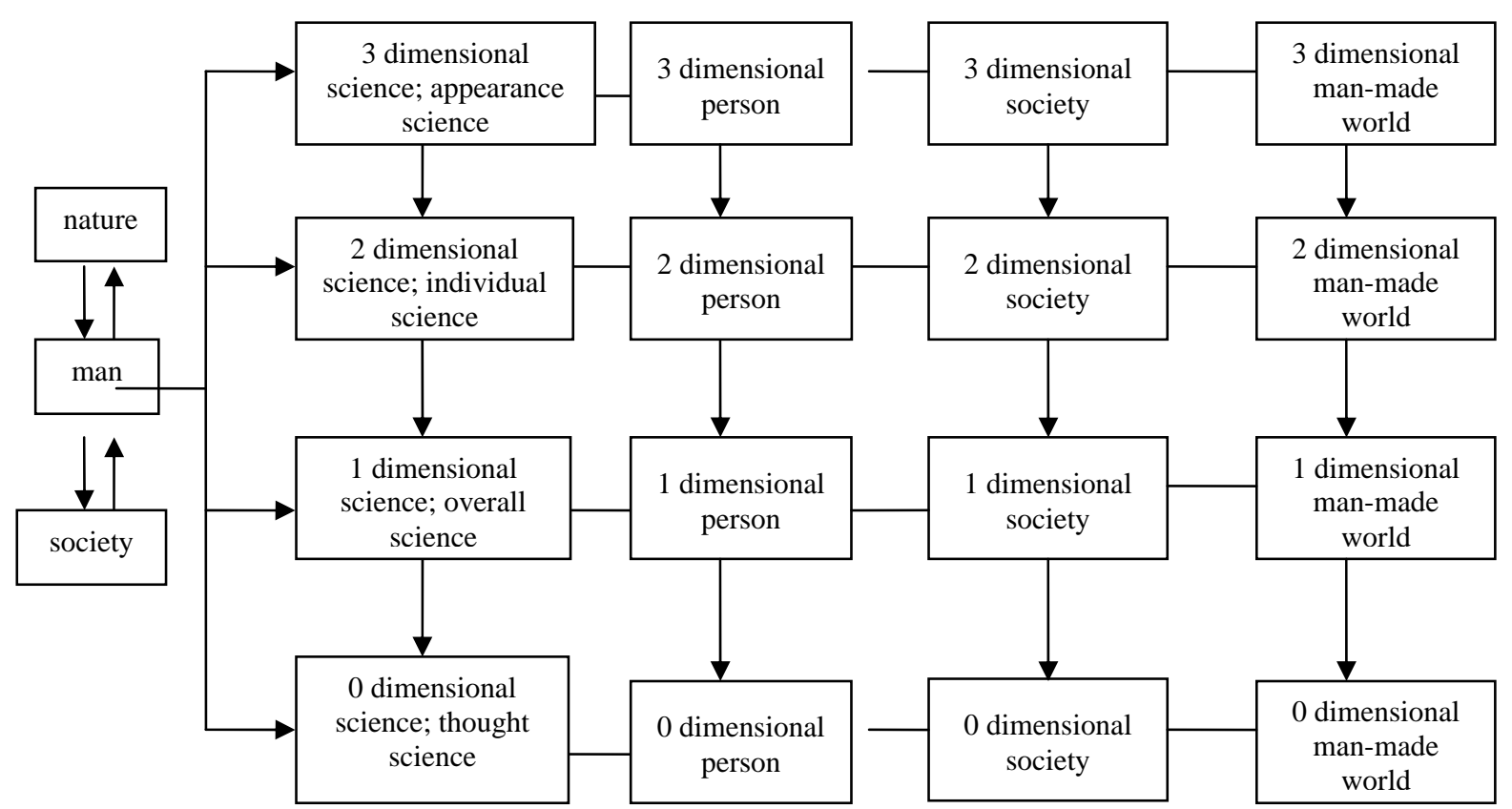

Fig. 2 Unification of man, nature, society and manmade worlds.

\subsection{The Four Seasonal Structure and Four Seasonal Movement of Science}

In four seasonal movement of the society, all existence of nature and society is understood by man and described as science and knowledge. Corresponding to Fig. 1, all science and knowledge form unified - dual four seasonal entire system of science and knowledge. This system includes the four seasons of natural science and technology and the four seasons of social science and technology. Each four seasonal structure includes the scientific discovery system, invention \& production system, consumption system, quality improvement system and control system.

\subsection{The Sublimation of Human Society Dimensions and Man-Made World Dimensions}

The driving force of the society is thought movement and development of science and technology. The development of science and technology pushes the development of human society and man-made world from 3 dimension, 2 dimension, towards 1 dimension and 0 dimension (Fig. 2).

\section{The $21^{\text {st }}$ Century is an Era of Unified Big Science: Era of Life Science \& Technology and Era of Thinking Science \& Technology}

With the combination of two major science \& technology from the East and West and the establishment of universal grand unification theory, mankind will enter the era of life science \& technology and era of cognitive science \& technology [6].

\section{Conclusions}

(1) The universe is life. The dominant movements in the universe are four seasonal movement and thought movement. The universe is unified in life and thought.

(2) The universal grand unified theory is a theory to completely unify all phenomena in the universe with a fundamental mode (structural mode, operational mode, generation mode and evolution mode).

(3) The book of changes of China: The great ultimate - two states - four images - eight hexagrams shows the fundamental mode that unifies the universe, which is the structural mode, operational mode, generation mode and evolution mode of cosmic life. 
The book of changes shows 1) life has four dimensions simultaneously, 0 dimensional, 1 dimensional, 2 dimensional and 3 dimensional states; 2) the breathing and four seasonal orbits of celestial bodies, whose movement characteristics are the elliptical orbits of celestial bodies; 3) three generation modes of life: generation by transformation, generation by augmentation, and self-generation. Different lives have different ways of generation. Different life forms are transforming into each other; 4) five major dynamic systems inside life; 5) the movement mode of sublimation/degration of cosmic life, the unification of theology and science as well as unification of religion and science; 6) four major dimensional and four seasonal classifications of the cosmos; 7) the derivation mode of life, i.e. one splits into two, and two integrate into one; 8) the unification of six major forces in the universe.

(4) Man has the power to understand the cosmos. The cognitive system of man has four seasonal structure and movement. The cognitive ability of man is divided into four dimensions: 3 dimension, 2 dimension, 1 dimension and 0 dimension. The Oriental science improves the level of cognitive organs and system on human body through cultivation to improve the understanding of the cosmos. Modern science understands the nature, man and society by the means of materialization of cognitive organs and systems.

(5) The universe is unified in life and thought. Galaxies in the universe are four seasonal entities. Small scale four seasonal celestial bodies participate in the four seasonal movements of large scale four seasonal celestial bodies. The universe is always in dynamic four seasonal equilibrium, and the interactions between all galaxies in the universe are bound to form the microwave background of the universe.

Life is inherent in the universe, so the question of the original of the universe and life simply does not exist. Just like living beings, celestial bodies can reproduce and living beings are the reproduction of celestial bodies.

Living beings on Earth sublimate/degrade with the overall movement of the solar system. When the solar system as a whole is in spring and summer, the inner energy of human body increases and when the solar system as a whole is in autumn and winter, the inner energy of human body drops.

(6) Man is a product of nature and the automatic organization of humans form society, form the four seasonal structure and four seasonal movement of the society, form the four seasonal structure and four seasonal movement of the science, and create physical worlds and spiritual worlds. Man, nature, society and man-made worlds are unified.

\section{References}

[1] Yan, J. Z. 2013. "Introduction to Unity of Physics and Biology, Inorganic Life Materials.” Journal of Physical Science and Application 3 (1): 58-64.

[2] An anonymous author. 2013. The Observation Notes by High-Level Qigong. Guilin: Guangxi normal university press, 12-430.

[3] Xiong, C. J. 2011. Explication of Thought of Lao Zi's People Following the Earth. Beijing: Tuanjie Press, 121-266.

[4] Fang, L. T. 2006. Buddhist Philosophy. Changchun: Changchun Press, 126-134.

[5] Xie, H. 2011. The Illustration Inner Canon of the Yellow Emperor. Beijing: TCM ancient books press, 398-472.

[6] Yan, J. Z. 2014. "Beginning of A New Revolution of Science \& Technology.” Journal of Physical Science and Application 4 (4): 269-275.

[7] Yan, J. Z. 2013. "Automatic Organization, Thinking Motion and Dynamics of the Universe." Journal of Physical Science and Application 3 (3) 199-208.

[8] Yang, L. 2012. The Book of Changes and Traditional Chinese Medicine. Beijing: Beijing Science and Technology Press, 1-9.

[9] Yan, J. Z. 2013. "State Space-Time and Four States of the Universe.” Journal of Physical Science and Application 3 (2) 127-134.

[10] Lu, T., and Luo, L. F. 2005. Comments on Substances: From Electrons to Quarks. Beijing: Science Press, 160-183.

[11] Yan, J. Z., and Yang, J. 2013. "Unity of Physics and Biology.” Materials Protection 46 (S2): 169-171.

[12] Lao Z. 2011. De Tao Ching. Beijing: The international 
cultural publishing company, 9-11.

[13] Xiong, C. J. 2011. Tao Medicine. Beijing: Tuanjin Press, 67-127.

[14] Tian, H. L. 2006. Life and 8 Gua. Taiyuan: Shanxi science and technology press, 148-150.

[15] Tian, H. L. 2006. The Original of The Book of Changes. Taiyuan: Shanxi Science and technology press, 66-94.

[16] Yan, J. Z. 2014. "The First Push of the Universe and Origin of Life.” Journal of Physical Science and
Application 4 (3): 198-205.

[17] Liang, W. H. 2012. Cell Biology. Beijing: Science press, 231-254.

[18] Niu, X., and Zhang, Z. X. 2012. Physiology. Beijing: China press of traditional Chinese medicine, 257-319.

[19] Xiong, C. J. 2011. Civilization and Education of Loong Culture. Beijing: Tuanjie Press, 80-1.

[20] Yan, J. Z. 2003. Zero Space-Time, Theory (Academic exchange data), Changsha, 291-306. 